\title{
Current Status of the Gasdynamic Mirror Fusion Propulsion Experiment
}

\author{
William J. Emrich, Jr. \\ NASA - Marshall Space Flight Center, Huntsville, Alabama 35812 \\ (256) 544-7504, bill.emrich@msfc.nasa.gov
}

\begin{abstract}
Nuclear fusion appears to be the most promising concept for producing extremely high specific impulse rocket engines. One particular fusion concept which seems to be particularly well suited for fusion propulsion applications is the gasdynamic mirror (GDM). An experimental GDM device has been constructed at the NASA Marshall Space Flight Center to provide an initial assessment of the feasibility of this type of propulsion system. An initial shakedown of the device is currently underway with initial experiments slated to occur in late 2001. This device would operate at much higher plasma densities and with much larger $L / D$ ratios than previous mirror machines. The high $L / D$ ratio minimizes to a large extent certain magnetic curvature effects which lead to plasma instabilities causing a loss of plasma confinement. The high plasma density results in the plasma behaving much more like a conventional fluid with a mean free path shorter than the length of the device. This characteristic helps reduce problems associated with "loss cone" microinstabilities. The device has been constructed to allow a considerable degree of flexibility in its configuration thus permitting the experiment to grow over time without necessitating a great deal of additional fabrication.
\end{abstract}

\section{INTRODUCTION}

One of the great deterrents to large scale exploration of the solar system has been and continues to be the tremendous cost associated with putting the massive amounts equipment and infrastructure into space to support such endeavors due to the relative low specific impulse available from chemical engines. Ideally, for solar system exploration, one would want a vehicle with specific impulses in the range of 10,000 to $200,000 \mathrm{~s}$ and $\alpha$ values of at least 10 (See Figure 1). Fusion engines, if they can be built in reasonable sizes, match these requirements quite closely and would be most suitable as the pitiuary propulsioni system for an interplanetary vehicle. Fusion based propulsion systems can also virtually eliminate mission window constraints effectively allowing unlimited manned exploration of the entire solar system (Emrich, 1996). Missions employing vehicles with fusion based main propulsion systems may begin and end at times convenient to meeting the mission objectives rather than being artificially constrained by launch window considerations. These vehicles also mitigate the need for large space infrastructures that would be required to support the transfer of massive amounts of fuel and supplies to lower a performing spacecraft.

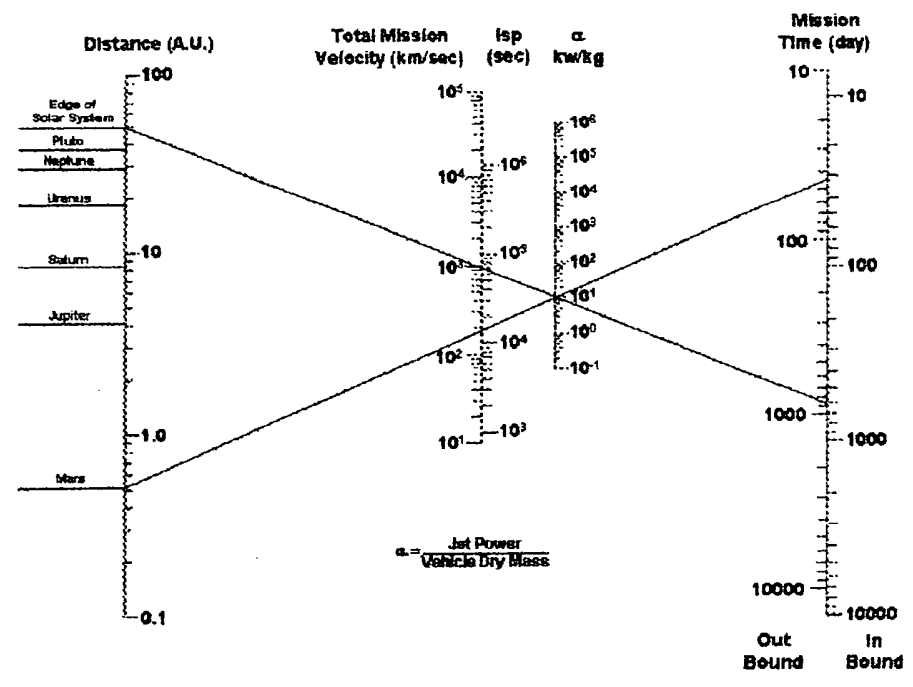

FIGURE 1: Optimized Planetary Round Trip Parametrics 
To be practical, a fusion engine must surpass a condition called "breakeven" wherein the energy resulting from fusion reactions is greater than that required initiate the reactions. Two conditions must be met to achieve breakeven. These conditions consist of high temperature $\left(>10^{8} \mathrm{~K}\right.$ ) and the Lawson Criteria (Density $\times$ Confinement Time $>10^{14}$ ion-sec/cc). Applying the Lawson criteria in different ways leads to radically different devices for producing fusion energy. High density and short confinement times yield inertial confinement systems while low density and long confinement times yield magnetic confinement systems.

\section{PRIOR DEVICES AND PROBLEMS}

One of the earliest types of magnetic confinement systems were mirror type systems. The largest mirror experiment in the U.S. was the Mirror Fusion Test Facility mod B (MFTF-B). This facility was completed in the early 80's but was never operated due to being canceled by DOE in favor of the mainline Tokamak effort (TFTR). This device was to have achieved near breakeven conditions. Several smaller mirror machines have been built across the country, but only one is currently operational (VASIMR). Mirror type fusion devices use open magnetic field line contours to confine charged particles in configurations called "magnetic wells". Mirror type "magnetic well" configurations are formed by constructing solenoid assemblies in which the magnetic fields at each end of the solenoid are stronger than that present near the center. Confinement is achieved in mirrors because of constraints on particle motion imposed by conservation of magnetic moment and of energy. Because of the open magnetic field line configuration of mirror fusion devices, they are particularly well suited for propulsion system applications since they allow for the easy ejection of plasma that is used to produce thrust.

In the past, there have been certain stability and power balance problems associated with mirror fusion devices that have led to their general abandonment as candidates for base commercial power generating applications. One type of instability is the Magnetohydrodynamic or MHD instability that is caused by large regions of "bad curvature" in the magnetic field lines. This type of instability leads to charge separation between the electrons and the ions causing uncontrollable ion drifts across the magnetic field lines to occur leading to a loss of confinement.

Another type of instability is called the "loss cone" microinstability. This type of instability is caused by velocity space asymmetries resulting from a depletion of the velocity space within the angle of the mirror "loss cone". Besides these instability problems, energy balances in traditional mirror configurations indicated that the " $Q$ " values (energy out/energy in) were only slightly greater than one. This low "Q" value resulted in unattractive commercial reactor designs and eventually led to the concept's general abandonment.

One type of mirror fusion device, however, seems to be capable of overcoming the stability and power balance problems mentioned above. This device is called the Gasdynamic Mirror or GDM (Kammash, 1995). The GDM overcomes MHD instabilities by having a very large length to diameter $(\mathrm{L} / \mathrm{D})$ ratio, minimizing the length of the device having bad magnetic field line curvature. In addition, since the instability is dependent upon the line integral of the plasma density in the regions of both good and bad field line curvature, the high plasma density in the mirror throat region of a mirror used for propulsive purposes compensates for the plasma in the relatively small regions of bad field line curvature.

The gasdynamic mirror overcomes the "loss cone" microinstability by having such a high plasma density that the plasma becomes quite collisional. This implies that the ion mean free path is much smaller than the length of the device. This collisionality causes any asymmetries in the ion velocity space to damp out quickly, thus suppressing this instability. The high plasma density also results in scaling relationships that indicate that the " $Q$ " values of the gasdynamic mirror should be somewhat more favorable than in conventional mirrors.

Presently, there exists two gasdynamic mirror fusion propulsion experiments. A medium sized gasdynamic mirror fusion device has been built and operated at the Novosibirsk Nuclear Physics Institute in Russia (Figure 2). The Russian GDM 
is approximately $9 \mathrm{~m}$ long and can achieve mirror magnetic field strengths of 16 tesla and central fields of 0.22 tesla. Stable plasmas with ion temperatures of about $100 \mathrm{eV}$ and ion densities of $10^{14} \mathrm{ion} / \mathrm{cc}$ have been achieved. The device is somewhat limited in that it can only operate in a transient decay mode and its physical layout cannot be easily reconfigured.

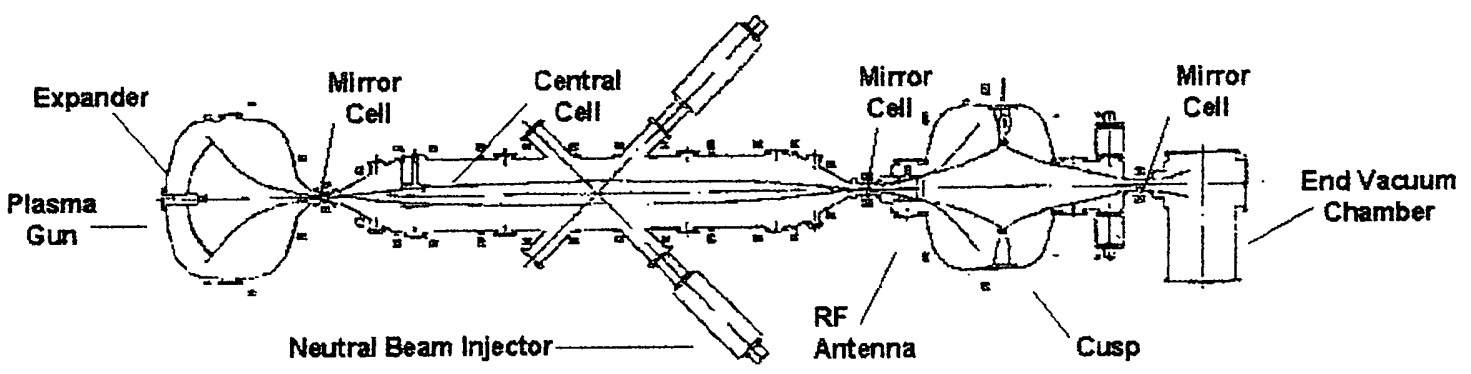

FIGURE 2. Russian Gasdynamic Mirror.

The other gasdynamic mirror (Figure 3) that is located at the NASA Marshall Space Flight Center is unlike the Russian device in that while it is currently smaller and lower powered, it is much more flexible from an operational standpoint and can very easily be reconfigured to accommodate changing experimental objectives.

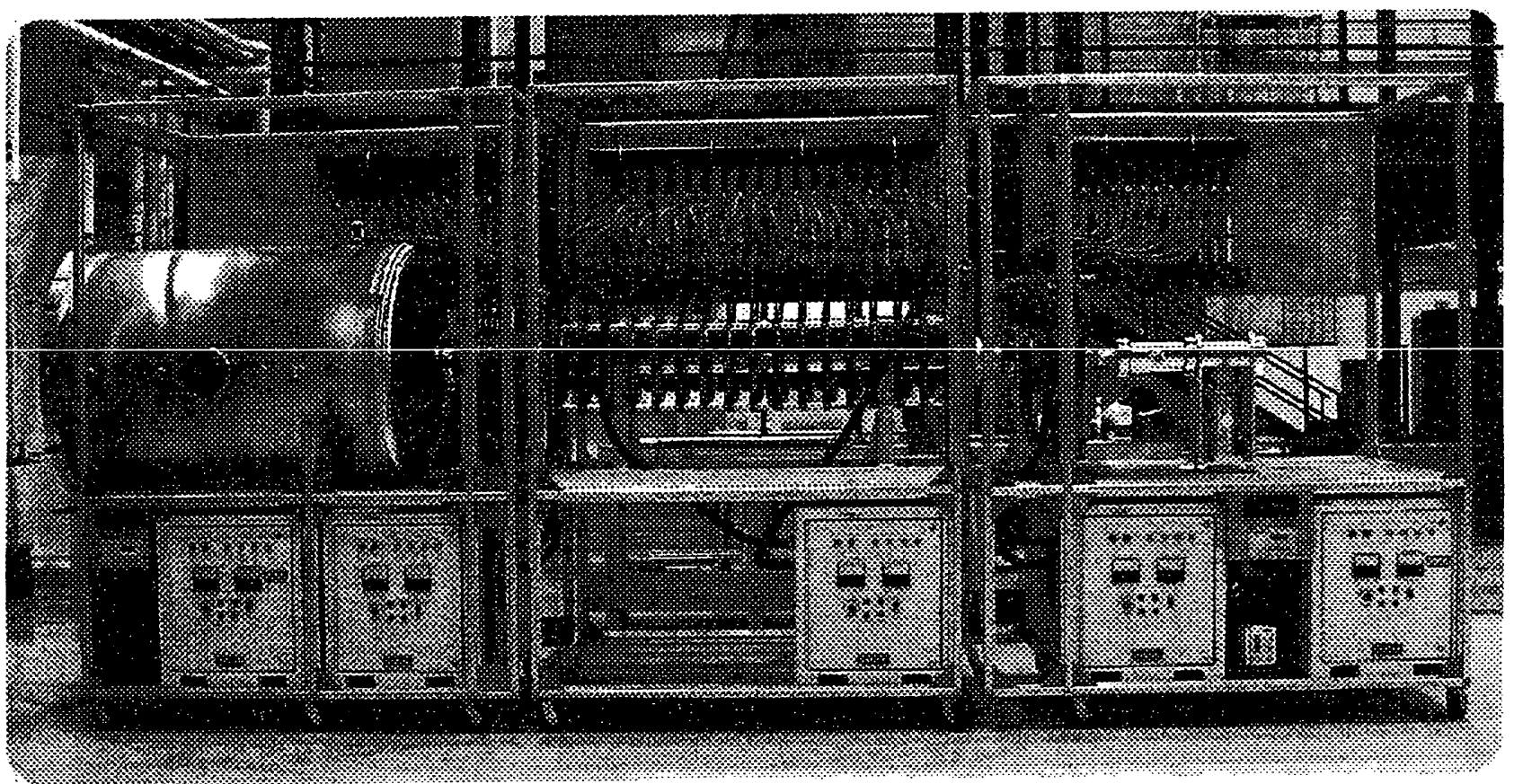

FIGURE 3. MSFC Gasdynamic Mirror.

Currently, the GDM is constructed in three segments: the vacuum chamber mirror plasma injector mirror segment, and the main plasma chamber segment. There are currently enough magnets and supporting structure to construct up to two additional main plasma chamber segments. The mirror segments themselves are also segmented such that the region between the mirror magnets and the main chamber can be expanded to accommodate new end plugging strategies without requiring the disassembly of the entire mirror segment. Table 1 enumerates the operating characteristics of the device. 
TABLE 1. GDM Experiment Characteristics.

\begin{tabular}{ll}
\hline Parameter & Value \\
\hline Length (m) & $3-7$ \\
Ion Temperature (eV) & $2-10$ \\
Injector Energy (eV) & 35 \\
Plasma Density (ion/cm ${ }^{3}$ ) & $10^{13}$ \\
Central Chamber Magnetic Field (tesla) & up to 0.33 \\
Mirror Magnetic Field (tesla) & up to 2.1 \\
Microwave Injector Power (w) & 1000 \\
Microwave Frequency (GHz) & 2.45 \\
\hline
\end{tabular}

The objective of the current research is to investigate the stability characteristics of the gasdynamic mirror and to map a region of parameter space within which the plasma can be confined in a stable steady state configuration. To this end, the mirror ratio, plasma density, and plasma " $\beta$ " will be varied over a range of values and measurements subsequently taken to determine the degree of plasma stability. These measurements will be compared to theoretical predictions of plasma stability under similar conditions. In order to prevent MHD flute instabilities from disrupting the plasma in the gasdynamic mirror, there must be sufficient plasma in the regions of "good" magnetic field curvature to offset the negative effects of the plasma in the regions of "bad" magnetic field curvature. This probably means that there is a mirror ratio limit beyond which the plasma becomes unstable as is illustrated in Figure 4. Where this limit occurs is of considerable importance with regard to the minimum practical size of the device since it has been shown that the length of the gasdynamic mirror is inversely proportional to the mirror ratio. In order to achieve the most compact propulsion system, therefore, one would want to operate the device at the highest possible mirror ratio consistent with plasma stability.

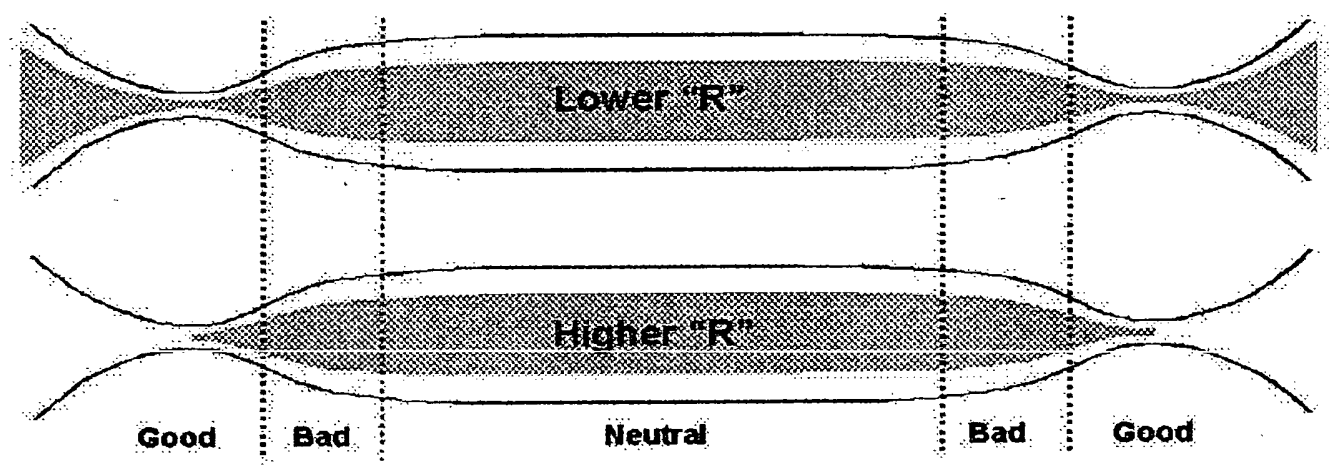

FIGURE 4. Instability Resulting from High Mirror Ratios.

A simple theory exists which will be used estimate limits of stability of the device. Since the MHD instabilities are primarily by the curvature of the magnetic field lines, the theory states that the change in the plasma internal energy along a field line must be greater than zero in order for the plasma to remain stable.

$$
\Delta E_{p}=C \int \frac{P_{\|}+P_{1}}{r R B^{2}} d l>0 .
$$

where: $\quad E_{p}=$ Internal energy of plasma

$\mathrm{r}=$ distance of magnetic field line from centerline of the GDM

$\mathrm{R}=$ magnetic field line radius of curvature

$\mathrm{P}=$ plasma pressure

$B=$ magnetic field strength 
The other major instability that will be studied in the gasdynamic mirror experiment is the loss cone microinstability. Basically, this instability is the result of an asymmetry in the ion velocity space distribution resulting from plasma loss through the mirror loss cone. If the plasma density is high enough, the mean free path of the ions will be sufficiently small so as to cause enough collisions to repopulate the velocity space depleted due to the escape of plasma through the mirror loss cone. In the GDM, the objective will be to create exactly this situation so that this instability will not occur under normal circumstances. For the first set of experiments, the GDM ion temperatures will only be in the order of $10 \mathrm{eV}$, therefore, ion densities of only $10^{11} \mathrm{ion} / \mathrm{cc}$ will be required to suppress this instability as is illustrated in Figure 5.

Plasma for the gasdynamic mirror is currently generated in a microwave cavity operated at $2.45 \mathrm{GHz}$ and located between the main magnets and the mirror magnets. Because of the magnetic field gradient present within the cavity, there can exist a point where the electrons in the working gas will resonate at a cyclotron frequency of $2.45 \mathrm{GHz}$. At this location the injected microwave energy will be absorbed resulting in intense electron heating and subsequent ionization. The hot electrons will flow along the field lines into the main chamber as a result of the magnetic field gradient resulting in a net negative charge in the main chamber and a net positive charge in the microwave cavity. The electric potential that occurs as a result of the above charge separation accelerates the ions in the cavity to fairly high energies through a process called ambipolar diffusion. Figure 6 illustrates the plasma generator in operation.

Currently, the experiment is completed and is undergoing an initial shakedown. Formal experiments will commence later this fall. The emphasis on the early experiments will be to establish a rough parameter space, outlining those regions where stable operation can be expected. Later experiments will be designed to refine and expand the region of stable operation.

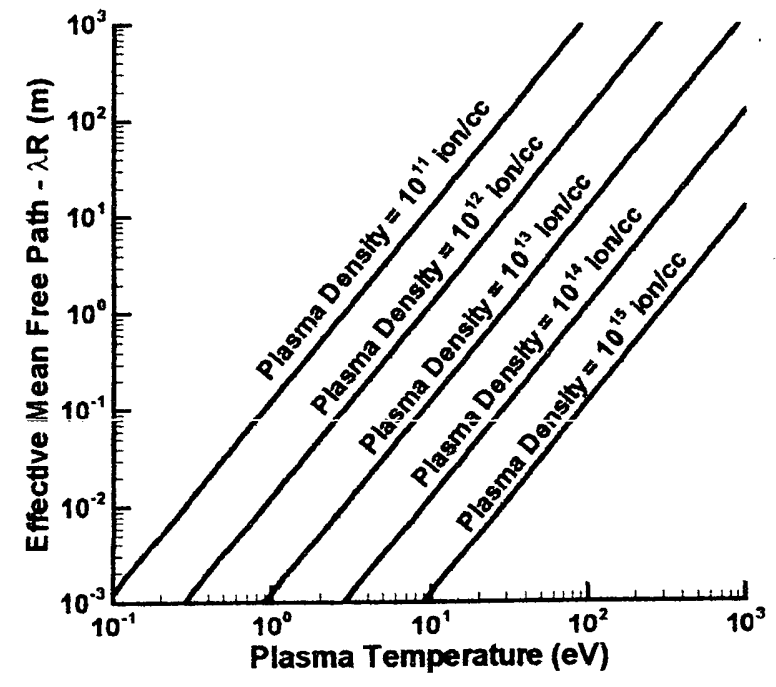

FIGURE 5. Ion Mean Free Paths.

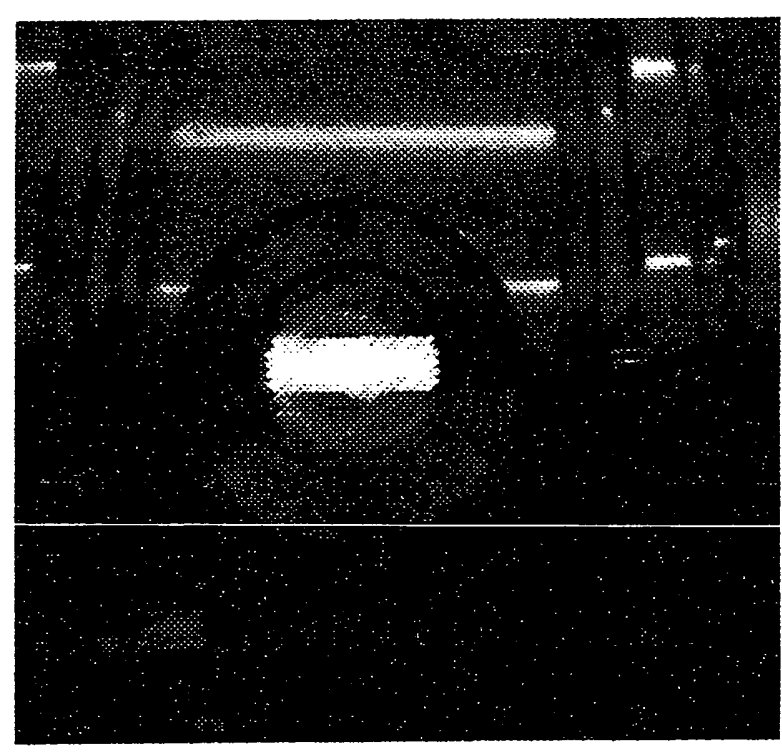

FIGURE 6. Gasdynamic Mirror Plasma Injector.

\section{SUMMARY}

Once operational later this year, the Gasdynamic Mirror experiment at MSFC will provide a test bed that may be used for performing experiments on a wide variety of technologies related to establishing the feasibility of various fusion propulsion concepts. For example, the large vacuum chamber allows for the testing of various magnetic nozzle configurations and segmented nature of the mirror segments allows for the incorporation of alternate end confinement devices such as Field Reversed Configurations for end plugging. It is hoped that the flexibility of this device will allow it to be useful long into the future for evaluating fusion propulsion related concepts. 


\section{ACKNOWLEDGMENTS}

The Gasdynamic Mirror experiment has been funded through generous internal support and through the Advanced Space Transportation Office. The support from many people at the NASA Marshall Space Flight Center has been crucial in bringing this experiment to fruition.

\section{REFERENCES}

Emrich, W. J., "Practical Interplanetary Travel Using A Gasdynamic Mirror Fusion Propulsion System," JointPropulsion Conference (1996), Paper \# AIAA 96-3067.

Kammash, T. and Lee, M., "Gasdynamic Fusion Propulsion System for Space Exploration," Journal of Propulsion and Power, Vol. 11, No. 3, May-June 1995, pp 544-553.

Emrich, W. J., "Gasdynamic Mirror Fusion Propulsion Experiment," STAIF (2001), pp 886-891 